\title{
Computational and analytical studies of the Randić index in Erdös-Rényi models
}

\author{
C. T. Martínez-Martínez ${ }^{\mathrm{a}}$, J. A. Méndez-Bermúdez ${ }^{\mathrm{a}, *}$, José M. Rodríguez ${ }^{\mathrm{b}}$, \\ José M. Sigarreta ${ }^{a, c}$ \\ ${ }^{a}$ Instituto de Física, Benemérita Universidad Autónoma de Puebla, Apartado Postal \\ J-48, Puebla 72570, Mexico \\ ${ }^{b}$ Universidad Carlos III de Madrid, Departamento de Matemáticas, Avenida de la \\ Universidad 30, 28911 Leganés, Madrid, Spain \\ ${ }^{c}$ Universidad Autónoma de Guerrero, Centro Acapulco CP 39610, Acapulco de Juárez, \\ Guerrero, Mexico
}

\begin{abstract}
In this work we perform computational and analytical studies of the Randić index $R(G)$ in Erdös-Rényi models $G(n, p)$ characterized by $n$ vertices connected independently with probability $p \in(0,1)$. First, from a detailed scaling analysis, we show that $\langle\bar{R}(G)\rangle=\langle R(G)\rangle /(n / 2)$ scales with the product $\xi \approx n p$, so we can define three regimes: a regime of mostly isolated vertices when $\xi<0.01(R(G) \approx 0)$, a transition regime for $0.01<\xi<10$ (where $0<R(G)<n / 2$ ), and a regime of almost complete graphs for $\xi>10$ $(R(G) \approx n / 2)$. Then, motivated by the scaling of $\langle\bar{R}(G)\rangle$, we analytically (i) obtain new relations connecting $R(G)$ with other topological indices and characterize graphs which are extremal with respect to the relations obtained and (ii) apply these results in order to obtain inequalities on $R(G)$ for graphs in Erdös-Rényi models.
\end{abstract}

Keywords: Randić index, vertex-degree-based topological index, random graphs, Erdös-Rényi graphs.

2000 MSC: 05C07, 05C80, 92E10

\footnotetext{
${ }^{*}$ Corresponding author

Email address: jmendezb@ifuap.buap.mx (J. A. Méndez-Bermúdez)
} 


\section{Introduction}

The interest in topological indices lies in the fact that they synthesize some of the fundamental properties of a molecule into a single value. With this in mind, several topological indices have been studied so far; it is worth noting the seminal work by Wiener (see [1]) in which he used the distances of a chemical graph in order to model properties of alkanes.

The Randić connectivity index was defined in [2] as

$$
R(G)=\sum_{u v \in E(G)} \frac{1}{\sqrt{d_{u} d_{v}}}
$$

where $u v$ denotes the edge of the graph $G$, and $d_{u}$ is the degree of the vertex $u$. Indeed, there are lots of works dealing with this index (see, e.g., [3, 44, 5]).

In [6, 7, 8], the first and second variable Zagreb indices are defined as

$$
M_{1}^{\alpha}(G)=\sum_{u \in V(G)} d_{u}^{\alpha}, \quad M_{2}^{\alpha}(G)=\sum_{u v \in E(G)}\left(d_{u} d_{v}\right)^{\alpha}
$$

with $\alpha \in \mathbb{R}$. The concept of variable molecular descriptors was proposed as a new way of characterizing heteroatoms in molecules (see [9, 10]). The essential idea is that the variables are determined during the regression; this allows to make the standard error of the estimate for a particular property (targeted in the study) as small as possible (see, e.g., [8]). The second variable Zagreb index is used in the structure-boiling point modeling of benzenoid hydrocarbons [11].

The general sum-connectivity index was defined in [12] as

$$
\chi_{\alpha}(G)=\sum_{u v \in E(G)}\left(d_{u}+d_{v}\right)^{\alpha} .
$$

Some relations of these indices are studied in ([13]).

In addition to the multiple applications of the Randic index in physical chemistry, this index has found several applications in other research areas and topics, such as information theory 14], network similarity [15], protein alignment [16], network heterogeneity [17], and network robustness [18]. Moreover, in [19] the concept of graph entropy for weighted graphs was introduced, especially the Randić weights. 
We want to recall that graphs have been widely used to study the properties of highly complex systems. Among them we can mention biological, social, and technological networks [20, 21]. Moreover, graphs can be classified as deterministic (regular and fractal) or disordered (random) [22]. Deterministic graphs follow specific construction rules, while in random graphs the parameters take fixed values but the graph itself has a random structure. In the later case a statistical study of graph ensembles with the same average properties must be performed, since the analysis of a single random graph is meaningless. There are well-known models of random graphs in the literature [23, 24], presumably the most popular are: the Erdös-Rényi model of random graphs, scale-free networks (introduced by Barabási and Albert), and smallworld networks (introduced by Watts and Strogatz). These three models have been extensively used to represent the organization of real-world complex systems (such as power grids or the Internet) through their underlying network structure [20, 23, 24].

Although random graph models are not able to predict some properties observed in real-world networks, such as nonvanishing clustering coefficient and power-law degree distributions [24], they have been deeply studied theoretically (e.g. 25]). In fact, several important results, such as the emergence of percolation, are analytically accesible from Erdös-Rényi graphs [23, 25]. Thus, here we consider Erdös-Rényi random graphs, which were proposed by Solomonoff and Rapoport [26] and investigated later in great detail by Erdős and Rényi [27, 28].

This work is organized as follows. First, in Sec. 2 we perform a detailed scaling analysis of the average Randić index to find its universal parameter, i.e., the parameter that statistically fixes the average value of $R(G)$. Then, in Sec. 3, we analytically (i) obtain new relations connecting $R(G)$ with other topological indices and (ii) apply these results in order to obtain inequalities on $R(G)$ for graphs in Erdös-Rényi models.

\section{Scaling analysis of the Randić index on Erdös-Rényi graphs}

We start with a computational (and statistical) study of the Randić index on Erdös-Rényi graphs. We consider random graphs $G$ from the standard Erdös-Rényi model $G(n, p)$, i.e., $G$ has $n$ vertices and each edge appears independently with probability $p \in(0,1)$. 
In Fig. 1(a) we show the average Randic index $\langle R(G)\rangle$ as a function of the probability $p$ of Erdös-Rényi graphs $G(n, p)$ of several orders $n$. Here, the average $\langle\cdot\rangle$ is computed over 2000 random graphs $G(n, p)$. We observe that the curves of $\langle R(G)\rangle$, for all the values of $n$ considered here, have a very similar shape as a function of $p:\langle R(G)\rangle$ shows a smooth transition (in log scale) from zero to $n / 2$ when $p$ increases from zero (isolated vertices) to one (complete graphs). Note that $n / 2$ is the maximal value that $R(G)$ can take.

Now, to ease our analysis, in Fig. 1(b) we present again $\langle R(G)\rangle$ but now normalized to $n / 2$ :

$$
\langle\bar{R}(G)\rangle=\frac{\langle R(G)\rangle}{n / 2} .
$$

From this figure we can clearly see that the main effect of increasing $n$ is the displacement of the curves $\langle\bar{R}(G)\rangle$ vs. $p$ to the left on the $p$-axis. Moreover, the fact that these curves, plotted in semi-log scale, are shifted the same amount on the $p$-axis when doubling $n$ make us anticipate the existence of a scaling parameter that depends on $n$. In order to search for that scaling parameter we first establish a measure to characterize the position of the curves $\langle\bar{R}(G)\rangle$ on the $p$-axis: We choose the value of $p$, that we label as $p^{*}$, for which $\langle\bar{R}(G)\rangle \approx 0.5$; see the dashed line in Fig. $1(\mathrm{~b})$. Notice that $p^{*}$ locates the transition point from isolated vertices to complete Erdös-Rényi graphs of size $n$.

Then, in Fig. 2(a) we plot $p^{*}$ versus $n$. The linear trend of the data (in log-log scale) in Fig. 2(a) suggests the power-law

$$
p^{*}=\mathcal{C} n^{\delta} .
$$

In fact, Eq. (3) provides an excellent fitting to the data with $\mathcal{C} \approx 0.77$ and $\delta \approx-1$. Therefore, by plotting again the curves of $\langle\bar{R}(G)\rangle$ now as a function of the probability $p$ divided by $p^{*}$,

$$
\xi \equiv \frac{p}{p^{*}} \propto \frac{p}{n^{\delta}} \approx \frac{p}{n^{-1}}=n p,
$$

we observe that curves for different graph sizes $n$ collapse on top of a single universal curve, see Fig. 2(b). This means that once the product $n p$ is fixed, the average Randić index on Erdös-Rényi graphs is also fixed. This statement is in accordance with the results reported in [29, 30], where the spectral and transport properties of Erdös-Rényi graphs where shown to be universal for the scaling parameter $n p$, see also [31, 32, 33]. 

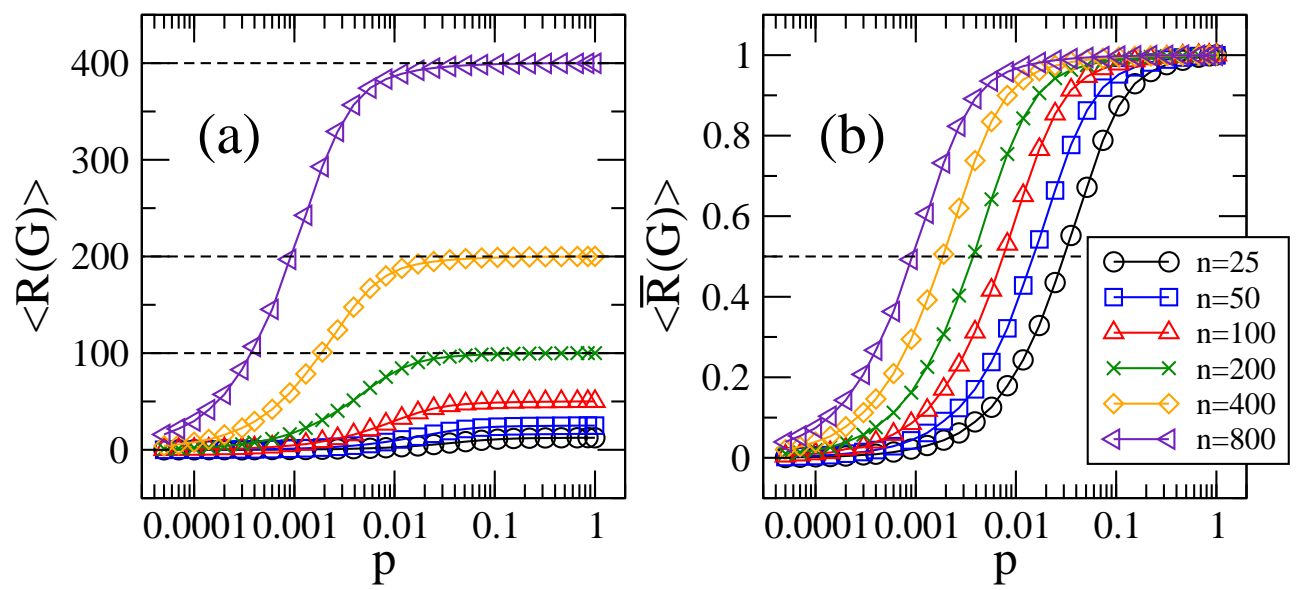

Figure 1: (a) Average Randić index $\langle R(G)\rangle$ as a function of the probability $p$ of ErdösRényi graphs $G(n, p)$ of different sizes $n \in[25,800]$. (b) $\langle R(G)\rangle$ normalized to $n / 2,\langle\bar{R}(G)\rangle$, as a function of $p$. Dashed lines in (a) indicate the values of $n / 2$ for $n \in[200,800]$. The dashed line in (b) indicates $\langle\bar{R}(G)\rangle=0.5$, used to define $p^{*}$. Each symbol was computed by averaging over 2000 random graphs $G(n, p)$.
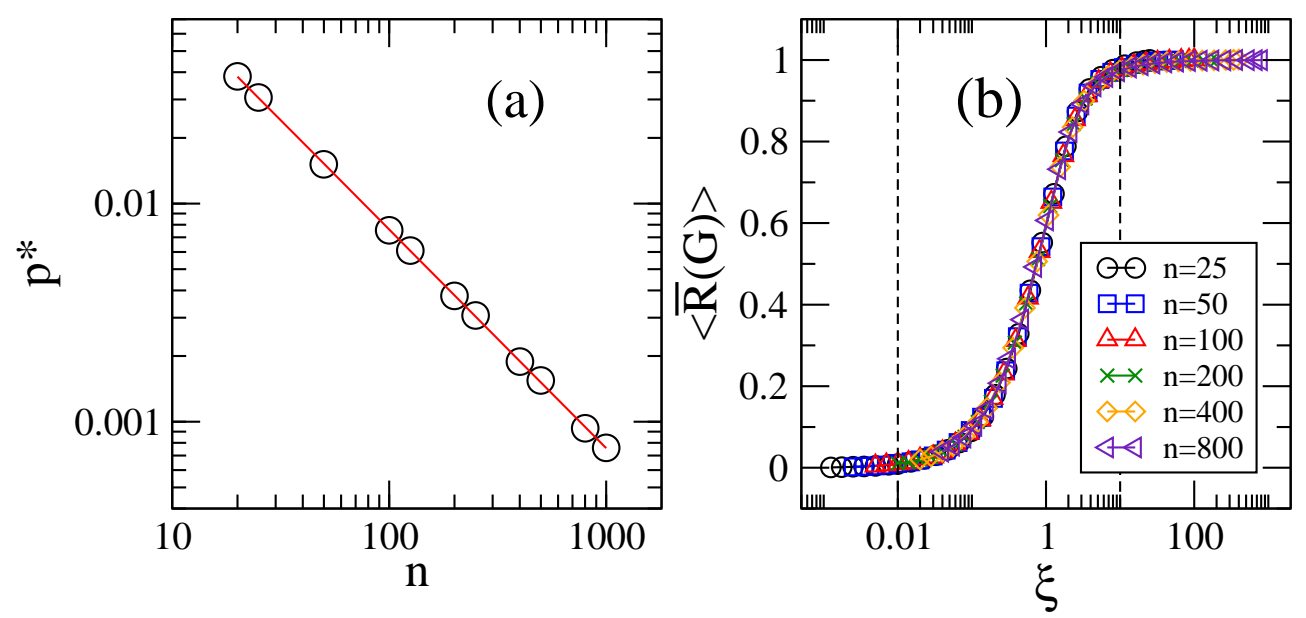

Figure 2: (a) $p^{*}$ (defined as the value of $p$ for which $\langle\bar{R}(G)\rangle \approx 0.5$ ) as a function of the graph size $n$. The red line is the fitting of Eq. (3) to the the data with fitting parameters $\mathcal{C}=0.76775$ and $\delta=-1.0021$. (b) $\langle\bar{R}(G)\rangle$ as a function of $\xi$. Vertical dashed lines in (b) indicate: The regime of mostly isolated vertices $(\xi<0.01)$, the transition regime $(0.01<\xi<10)$, and the regime of almost complete graphs $(\xi>10)$. 

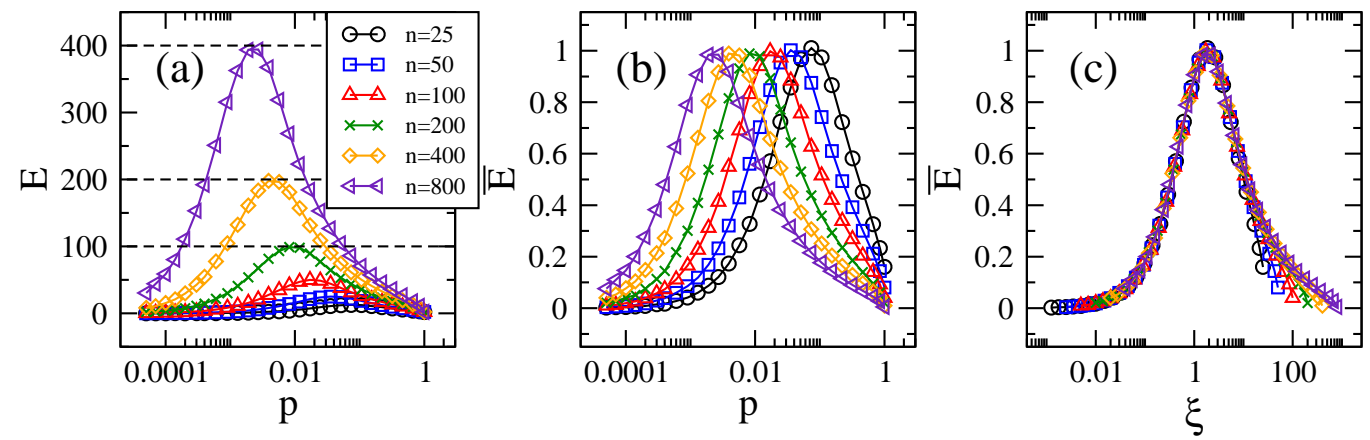

Figure 3: (a) Randić Matrix energy $E$ as a function of the probability $p$ for Erdös-Rényi graphs of size $n$. Dashed lines indicate the values of $n / 2$ for $n \in[200,800]$. (b) $\bar{E}=$ $E /(n / 2)$ as a function $p$. (c) $\bar{E}$ as a function $\xi$.

Additionally, from our previous experience, see e.g., [29, 30, 31, 32, 33], we expect that other quantities related to $R(G)$ will also be scaled with $\xi$. Indeed, we validate this conjecture by analyzing the energy $E(n, p)$ of the Erdös-Rényi graphs $G(n, p)$ defined as [34, 35]

$$
E(n, p)=\sum_{i=1}^{n}\left|e_{i}\right|,
$$

where $e_{i}$ are the eigenvalues of the corresponding Randić matrix [34, 35]:

$$
R_{i j}= \begin{cases}\left(d_{i} d_{j}\right)^{-1 / 2} & \text { if } v_{i} \sim v_{j}, \\ 0 & \text { otherwise. }\end{cases}
$$

Thus in Fig. 3(a) we present the energy $E$ as a function of the probability $p$ of Erdös-Rényi graphs of several sizes $n$. The curves $E$ vs. $p$ show a similar behavior for different values of $n$ : For small $p, E$ increases with $p$ until it reaches $n / 2$ (the maximum value it can take), then $E$ decreases from its maximum by further increasing $p$ giving to the curves $E$ vs. $p$ a bell-like shape in $\log$ scale. Now, for convenience, we normalize $E$ to $n / 2$ (that we name $\bar{E}$ ) and plot it in Fig. 3(b). Here it is clear that the curves $\bar{E}$ vs. $p$ are very similar but shifted to the left on the $p$-axis for increasing $n$. Finally, in Fig. 3(1) we plot $\bar{E}$ as a function of the scaling parameter $\xi$, see Eq. (4), and show that all curves fall one on top of the other (except for finite size effects at large $\xi$ ). Therefore, we confirm that the energy of Erdös-Rényi graphs (as defined in Eq. (5)) also scales with the parameter $\xi$; that is, once $\xi$ is fixed 


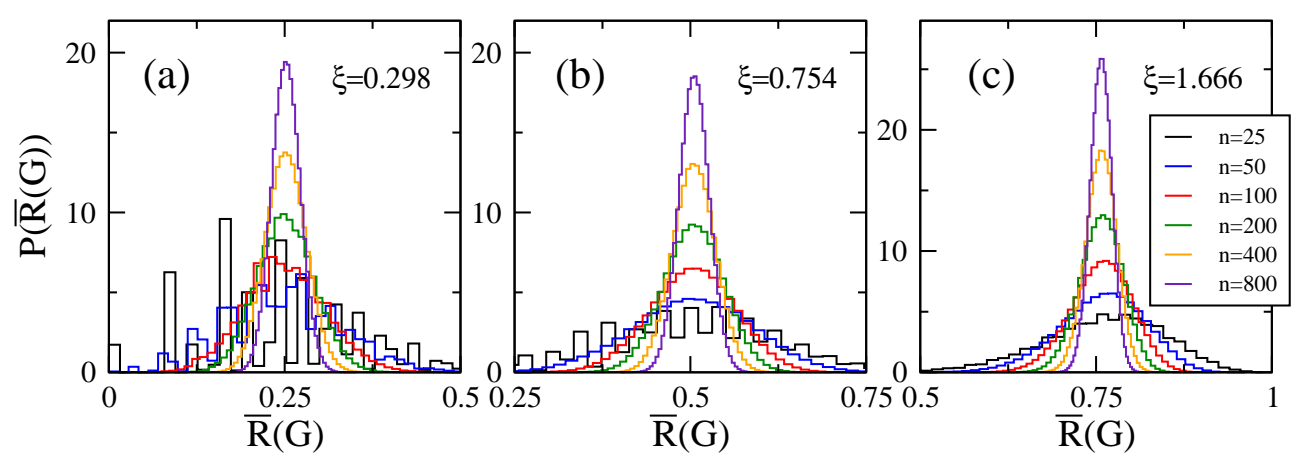

Figure 4: Probability distribution functions of $\bar{R}(G), P(\bar{R}(G))$, for several graph sizes $n$ at fixed values of $\langle\bar{R}(G)\rangle$ : (a) $\langle\bar{R}(G)\rangle=0.25$, (b) $\langle\bar{R}(G)\rangle=0.5$, and (c) $\langle\bar{R}(G)\rangle=0.75$. The corresponding values of $\xi$ are given in the panels. Each histogram was constructed with 2000 values of $\bar{R}(G)$.

the normalized energy $\bar{E}$ is (statistically) the same for different parameter combinations $(n, p)$. Additionally, from Fig. 3(1) we can conclude that the maximum value of $E$ occurs in the interval $1<\xi<2$, in close agreement with the delocalization transition value for the eigenvectors of Erdös-Rényi graphs reported in [29, 36, 37, 38, 39] to be $\xi \approx 1.4$.

Even though we have shown that $\xi$ scales both $\langle\bar{R}(G)\rangle$ and $\bar{E}$ reasonably well, it is fair to say that there are additional quantities related to $\bar{R}(G)$ which are still size dependent for fixed $\xi$. See for example Fig. 4, where we show probability distribution functions of $\bar{R}(G)$ at fixed $\xi$. In this figure we observe that, even for fixed $\xi$ (or equivalently, for fixed $\langle\bar{R}(G)\rangle$ ), $P(\bar{R}(G)$ ) becomes narrower for increasing $n$. This means that the variance and the minimal and maximal values of $\bar{R}(G)$ change with $n$, as can be clearly seen in Fig. 5. This motivate us to look for bounds and inequalities on the Randić index on Erdös-Rényi graphs, which is the main topic of the following Section.

\section{Inequalities for the Randić index on Erdös-Rényi models}

We recall that we consider a Random Graph $G$ from the standard ErdösRényi model $G(n, p)$. In the following, $G$ denotes a finite simple graph such that each connected component of $G$ has, at least, one edge (there are no isolated vertices). We say that a statement holds for almost every graph if the probability of the set of graphs for which the statement fails tends to 0 as $n \rightarrow \infty$. 

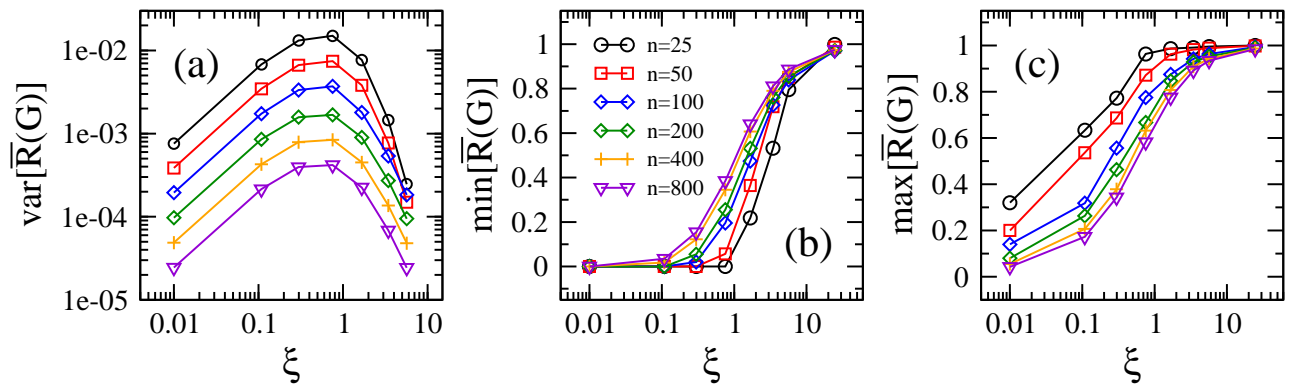

Figure 5: (a) $\operatorname{var}[\bar{R}(G)]$, (b) $\min [\bar{R}(G)]$, and (c) $\max [\bar{R}(G)]$ as a function of $\xi$. Each symbol was computed from 2000 values of $\bar{R}(G)$.

The following facts about the Erdös-Rényi model are well-known [40] (see also [41]):

(1) Almost every graph $G$ has $m=p n(n-1) / 2+o\left(n^{2}\right)$ edges.

(2) Almost every graph $G$ has maximum degree $\Delta=p(n-1)+(2 p q n \log n)^{1 / 2}+$ $o\left((n \log n)^{1 / 2}\right)$, with $p \in[1 / 2,1)$ and $q=1-p$.

(3) Almost every graph $G$ has minimum degree $\delta=q(n-1)-(2 p q n \log n)^{1 / 2}+$ $o\left((n \log n)^{1 / 2}\right)$, with $p \in[1 / 2,1)$ and $q=1-p$.

In the previous equalities we are using Landau's notation: Recall that $f(n)=g(n)+o(a(n))$ means that

$$
\lim _{n \rightarrow \infty} \frac{f(n)-g(n)}{a(n)}=0
$$

and $f(n)=g(n)+O(a(n))$ means that

$$
\frac{f(n)-g(n)}{a(n)}
$$

is a bounded sequence.

The following result relates the Randić and the (-2)-sum-connectivity indices.

Theorem 1. Let $G$ be a graph with minimum degree $\delta$ and maximum degree $\Delta$. Then 


$$
\begin{array}{ll}
4 \delta \chi_{-2}(G) \leq R(G) \leq 4 \Delta \chi_{-2}(G), & \text { if } \delta / \Delta \geq t_{0}, \\
4 \delta \chi_{-2}(G) \leq R(G) \leq \frac{(\Delta+\delta)^{2}}{\sqrt{\Delta \delta}} \chi_{-2}(G), & \text { if } \delta / \Delta<t_{0},
\end{array}
$$

where $t_{0}$ is the unique solution of the equation $t^{3}+5 t^{2}+11 t-1=0$ in the interval $(0,1)$. The equality in the lower bound is attained if and only if $G$ is regular. The equality in the first upper bound is attained if and only if $G$ is regular; the equality in the second upper bound is attained if and only if $G$ is a biregular graph.

Proof. Since $a(t)=t^{3}+5 t^{2}+11 t-1$ is an increasing function on the interval $[0,1], a(0)<0$ and $a(1)>0$, there exists a unique solution of the equation $t^{3}+5 t^{2}+11 t-1=0$ in the interval $(0,1)$. Hence, the number $t_{0}$ is well-defined.

Let us compute the maximum and minimum values of the function $g$ : $[\delta, \Delta] \times[\delta, \Delta] \rightarrow \mathbb{R}$ given by

$$
g(x, y)=\frac{\sqrt{x y}}{(x+y)^{2}}
$$

Since $g(x, y)=g(y, x)$, we can assume that $x \leq y$. The partial derivatives of $g$ are

$$
\begin{aligned}
\frac{\partial g}{\partial x}(x, y) & =\frac{x^{-1 / 2} y^{1 / 2}(x+y)-4 x^{1 / 2} y^{1 / 2}}{2(x+y)^{3}} \\
& =x^{-1 / 2} y^{1 / 2} \frac{y-3 x}{2(x+y)^{3}}, \\
\frac{\partial g}{\partial y}(x, y) & =y^{-1 / 2} x^{1 / 2} \frac{x-3 y}{2(x+y)^{3}}
\end{aligned}
$$

Since $y \geq x \geq \delta>0$, we obtain $\partial g / \partial y<0$ and $g$ is a decreasing function on $y$. Therefore, $g$ attains its minimum value on $\{(x, \Delta) \mid \delta \leq x \leq \Delta\}$ and its maximum value on $\{(x, x) \mid \delta \leq x \leq \Delta\}$. Note that $g(x, x)=1 /(4 x) \leq$ $1 /(4 \delta)$.

If $\Delta \leq 3 \delta$, then $\partial g / \partial x(x, \Delta)<0$ for every $x>\delta$.

If $\Delta>3 \delta$, then $\partial g / \partial x(x, \Delta)>0$ for every $\delta \leq x<\Delta / 3$ and $\partial g / \partial x(x, \Delta)<$ 0 for every $\Delta / 3<x \leq \Delta$. 
Hence, we have in every case

$$
\min \left\{\frac{1}{4 \Delta}, \frac{\sqrt{\Delta \delta}}{(\Delta+\delta)^{2}}\right\}=\min \{g(\Delta, \Delta), g(\delta, \Delta)\} \leq g(x, y) \leq \frac{1}{4 \delta} .
$$

Thus,

$$
\begin{aligned}
& \min \left\{\frac{1}{4 \Delta}, \frac{\sqrt{\Delta \delta}}{(\Delta+\delta)^{2}}\right\} \frac{1}{\sqrt{d_{u} d_{v}}} \leq \frac{1}{\left(d_{u}+d_{v}\right)^{2}} \leq \frac{1}{4 \delta} \frac{1}{\sqrt{d_{u} d_{v}}} \\
& \min \left\{\frac{1}{4 \Delta}, \frac{\sqrt{\Delta \delta}}{(\Delta+\delta)^{2}}\right\} R(G) \leq \chi_{-2}(G) \leq \frac{1}{4 \delta} R(G)
\end{aligned}
$$

If the equality in the lower bound is attained, then $\left(d_{u}, d_{v}\right)=(\delta, \delta)$ for all $u v \in E(G)$; hence, $d_{u}=\delta$ for all $u \in V(G)$ and so, $G$ is regular.

In order to prove the upper bounds, it suffices to show that the inequality

$$
\frac{1}{4 \Delta} \leq \frac{\sqrt{\Delta \delta}}{(\Delta+\delta)^{2}}
$$

holds if and only if $\delta / \Delta \geq t_{0}$.

Inequality (7) is equivalent to the following statements

$$
\begin{array}{ll}
(\Delta+\delta)^{2} \leq 4 \Delta \sqrt{\Delta \delta}, & \left(1+\frac{\delta}{\Delta}\right)^{2} \leq 4 \sqrt{\frac{\delta}{\Delta}} \\
\left(1+\frac{\delta}{\Delta}\right)^{4} \leq 16 \frac{\delta}{\Delta}, & \frac{\delta^{4}}{\Delta^{4}}+4 \frac{\delta^{3}}{\Delta^{3}}+6 \frac{\delta^{2}}{\Delta^{2}}-12 \frac{\delta}{\Delta}+1 \leq 0 .
\end{array}
$$

Since $0<\delta / \Delta \leq 1$, let us consider the function $b(t)=t^{4}+4 t^{3}+6 t^{2}-12 t+1$ for $t \in(0,1]$. Since $b(t)=(t-1)\left(t^{3}+5 t^{2}+11 t-1\right)=(t-1) a(t)$, we have $a(t) \leq 0$ if and only if $t \in\left[t_{0}, 1\right]$. Hence, inequality (7) holds if and only if $\delta / \Delta \geq t_{0}$. Since the coefficients of the polynomial $a(t)=t^{3}+5 t^{2}+11 t-1$ are rational numbers, and the coefficients of $t^{3}$ and $t^{0}$ of the polynomial $a(t)$ are 1 and -1 , respectively, we have that $t_{0} \notin \mathbb{Q}$. Note that this condition is equivalent to $\delta / \Delta>t_{0}$, since $t_{0} \notin \mathbb{Q}$; therefore, the equality in (17) is attained if and only if $\delta=\Delta$.

Therefore, the upper bounds hold.

If $\delta / \Delta \geq t_{0}$, then the previous argument gives that $f$ attains its minimum value just at the point $(\Delta, \Delta)$. Thus, the equality in the upper bound is 
attained if and only if $\left(d_{u}, d_{v}\right)=(\Delta, \Delta)$ for every $u v \in E(G)$, i.e., $G$ is regular.

If $\delta / \Delta<t_{0}$, then $f$ attains its minimum value just at the points $(\delta, \Delta)$ and $(\Delta, \delta)$. Hence, the equality in the upper bound is attained if and only if $\left\{d_{u}, d_{v}\right\}=\{\delta, \Delta\}$ for every $u v \in E(G)$, i.e., $G$ is biregular. In this case, $G$ can not be regular since $\delta<t_{0} \Delta<\Delta$.

Theorem 1 have the following consequence on Random Graphs.

Corollary 2. In the Erdös-Rényi model $G(n, p)$, with $p \in[1 / 2,1)$ and $q=$ $1-p$, almost every graph $G$ satisfies

$$
4 q n+O\left((n \log n)^{1 / 2}\right) \leq \frac{R(G)}{\chi_{-2}(G)} \leq \max \left\{4 p, \frac{1}{\sqrt{p q}}\right\} n+O\left((n \log n)^{1 / 2}\right) .
$$

Proof. The conclusion in Theorem 1 can be written as follows:

$$
4 \delta \leq \frac{R(G)}{\chi_{-2}(G)} \leq \max \left\{4 \Delta, \frac{(\Delta+\delta)^{2}}{\sqrt{\Delta \delta}}\right\} .
$$

Thus, the first inequality is a direct consequence of (8) and (3). Let us prove the second one. Items (2) and (3) give for almost every graph

$$
\begin{aligned}
\frac{(\Delta+\delta)^{2}}{\sqrt{\Delta \delta}} & =\frac{\left(n+o\left((n \log n)^{1 / 2}\right)\right)^{2}}{\sqrt{p q n^{2}+O\left(n(n \log n)^{1 / 2}\right)}}=\frac{n^{2}+o\left(n(n \log n)^{1 / 2}\right)}{\sqrt{p q} n+O\left((n \log n)^{1 / 2}\right)} \\
& =\frac{n}{\sqrt{p q}}\left(1-\frac{O\left((n \log n)^{1 / 2}\right)}{\sqrt{p q} n}\right)+\frac{o\left(n(n \log n)^{1 / 2}\right)}{\sqrt{p q} n+O\left((n \log n)^{1 / 2}\right)} \\
& =\frac{n}{\sqrt{p q}}+O\left((n \log n)^{1 / 2}\right)+o\left((n \log n)^{1 / 2}\right) \\
& =\frac{n}{\sqrt{p q}}+O\left((n \log n)^{1 / 2}\right) .
\end{aligned}
$$

This fact, (86) and item (2) give the second inequality for almost every graph.

Corollary 2 has the following consequence.

Corollary 3. In the Erdös-Rényi model $G(n, p)$, with $p=1 / 2$, almost every graph $G$ satisfies

$$
\frac{R(G)}{\chi_{-2}(G)}=2 n+O\left((n \log n)^{1 / 2}\right) \text {. }
$$


The following technical result appears in [42, Corollary 2.3].

Lemma 4. Let $g$ be the function $g(x, y)=2 \sqrt{x y} /(x+y)$ with $0<a \leq x, y \leq$ b. Then

$$
\frac{2 \sqrt{a b}}{a+b} \leq g(x, y) \leq 1 \text {. }
$$

Given a graph $G$, let us define

$$
\delta_{G}=\min _{u v \in E(G)} \frac{2 \sqrt{d_{u} d_{v}}}{d_{u}+d_{v}}, \quad \Delta_{G}=\min _{u v \in E(G)} \frac{2 \sqrt{d_{u} d_{v}}}{d_{u}+d_{v}} .
$$

Let $G$ be a graph with maximum degree $\Delta$ and minimum degree $\delta$. Then Lemma 4 gives, for every $u v \in E(G)$,

$$
\frac{2 \sqrt{\Delta \delta}}{\Delta+\delta} \leq \delta_{G} \leq \frac{2 \sqrt{d_{u} d_{v}}}{d_{u}+d_{v}} \leq \Delta_{G} \leq 1
$$

Since

$$
\delta_{G} \leq \frac{2 d_{u} d_{v}}{\left(d_{u}+d_{v}\right) \sqrt{d_{u} d_{v}}} \leq \Delta_{G}
$$

for every $u v \in E(G)$, we obtain

$$
\frac{\delta_{G}}{2} \frac{d_{u}+d_{v}}{d_{u} d_{v}} \leq \frac{1}{\sqrt{d_{u} d_{v}}} \leq \frac{\Delta_{G}}{2} \frac{d_{u}+d_{v}}{d_{u} d_{v}} .
$$

For every function $f$, we have

$$
\sum_{u v \in E(G)}\left(f\left(d_{u}\right)+f\left(d_{u}\right)\right)=\sum_{u \in V(G)} d_{u} f\left(d_{u}\right)
$$

and so,

$$
\sum_{u v \in E(G)} \frac{d_{u}+d_{v}}{d_{u} d_{v}}=\sum_{u v \in E(G)}\left(\frac{1}{d_{u}}+\frac{1}{d_{v}}\right)=\sum_{u \in V(G)} d_{u} \frac{1}{d_{u}}=\sum_{u \in V(G)} 1=n .
$$

This equality and (10) give the inequalities:

$$
\frac{n \delta_{G}}{2} \leq R(G) \leq \frac{n \Delta_{G}}{2}
$$


A similar result is proved in [41]; there, the author uses an argument based on differential calculus.

As a consequence of the previous result and (9), we obtain the known inequalities

$$
\frac{\sqrt{\Delta \delta}}{\Delta+\delta} n \leq R(G) \leq \frac{n}{2} .
$$

Notice that the right inequality has already been computationally verified in Fig. 1

Proposition 5. In the Erdös-Rényi model $G(n, p)$, with $p \in[1 / 2,1)$ and $q=1-p$, almost every graph $G$ satisfies

$$
R(G) \geq \sqrt{p q} n+O\left((n \log n)^{1 / 2}\right) .
$$

Proof. Let us consider the Erdös-Rényi model $G(n, p)$. Almost every graph $G$ satisfies

$$
\begin{aligned}
\frac{\sqrt{\Delta \delta}}{\Delta+\delta} n & =\frac{\sqrt{p q n^{2}+O\left(n(n \log n)^{1 / 2}\right)}}{n+o\left((n \log n)^{1 / 2}\right)} n=\frac{\sqrt{p q} n+O\left((n \log n)^{1 / 2}\right)}{n+o\left((n \log n)^{1 / 2}\right)} n \\
& =\sqrt{p q} n\left(1-\frac{o\left((n \log n)^{1 / 2}\right)}{n}\right)+\frac{O\left((n \log n)^{1 / 2}\right)}{n+o\left((n \log n)^{1 / 2}\right)} n \\
& =\sqrt{p q} n+o\left((n \log n)^{1 / 2}\right)+O\left((n \log n)^{1 / 2}\right) \\
& =\sqrt{p q} n+O\left((n \log n)^{1 / 2}\right) .
\end{aligned}
$$

This fact and (11) allow to obtain the result.

Corollary 6. In the Erdös-Rényi model $G(n, p)$, with $p=1 / 2$, almost every graph $G$ satisfies

$$
R(G)=\frac{n}{2}+O\left((n \log n)^{1 / 2}\right) .
$$

In fact, this Corollary has already been computationally verified in Fig. 1.

Proposition 7. Let $G$ be a graph with $n$ vertices, minimum degree $\delta$ and maximum degree $\Delta$. Then

$$
\begin{aligned}
& \frac{n}{2}-\frac{1}{2 \delta^{2}}\left(M_{1}(G)-2 M_{2}^{1 / 2}(G)\right) \leq R(G) \leq \frac{n}{2}-\frac{1}{2 \Delta^{2}}\left(M_{1}(G)-2 M_{2}^{1 / 2}(G)\right), \\
& \frac{1}{2 \Delta^{2}}\left(M_{1}(G)+2 M_{2}^{1 / 2}(G)\right)-\frac{n}{2} \leq R(G) \leq \frac{1}{2 \delta^{2}}\left(M_{1}(G)+2 M_{2}^{1 / 2}(G)\right)-\frac{n}{2} .
\end{aligned}
$$

The equality is attained in each bound if and only if $G$ is a regular graph. 
Proof. In the argument in the proof of [43, Theorem 1] appears the following relation:

$$
R(G)=\frac{n}{2}-\frac{1}{2} \sum_{u v \in E(G)} \frac{\left(\sqrt{d_{u}}-\sqrt{d_{v}}\right)^{2}}{d_{u} d_{v}}
$$

and we deduce

$$
\frac{n}{2}-\frac{1}{2 \delta^{2}} \sum_{u v \in E(G)}\left(\sqrt{d_{u}}-\sqrt{d_{v}}\right)^{2} \leq R(G) \leq \frac{n}{2}-\frac{1}{2 \Delta^{2}} \sum_{u v \in E(G)}\left(\sqrt{d_{u}}-\sqrt{d_{v}}\right)^{2} .
$$

Since

$$
\sum_{u v \in E(G)}\left(\sqrt{d_{u}}-\sqrt{d_{v}}\right)^{2}=\sum_{u v \in E(G)}\left(d_{u}+d_{v}\right)-2 \sum_{u v \in E(G)} \sqrt{d_{u} d_{v}}=M_{1}(G)-2 M_{2}^{1 / 2}(G),
$$

we obtain the first and second inequalities.

Since

$$
\begin{aligned}
-n+\sum_{u v \in E(G)} \frac{\left(\sqrt{d_{u}}+\sqrt{d_{v}}\right)^{2}}{d_{u} d_{v}}=-\sum_{u \in V(G)} d_{u} \frac{1}{d_{u}}+\sum_{u v \in E(G)} \frac{\left(\sqrt{d_{u}}+\sqrt{d_{v}}\right)^{2}}{d_{u} d_{v}} \\
=-\sum_{u v \in E(G)}\left(\frac{1}{d_{u}}+\frac{1}{d_{v}}\right)+\sum_{u v \in E(G)} \frac{d_{u}+d_{v}+2 \sqrt{d_{u} d_{v}}}{d_{u} d_{v}} \\
=-\sum_{u v \in E(G)} \frac{d_{u}+d_{v}}{d_{u} d_{v}}+\sum_{u v \in E(G)} \frac{d_{u}+d_{v}+2 \sqrt{d_{u} d_{v}}}{d_{u} d_{v}} \\
=\sum_{u v \in E(G)} \frac{2 \sqrt{d_{u} d_{v}}}{d_{u} d_{v}}=2 R(G),
\end{aligned}
$$

we have

$$
\begin{gathered}
R(G)=-\frac{n}{2}+\frac{1}{2} \sum_{u v \in E(G)} \frac{\left(\sqrt{d_{u}}+\sqrt{d_{v}}\right)^{2}}{d_{u} d_{v}}, \\
-\frac{n}{2}+\frac{1}{2 \Delta^{2}} \sum_{u v \in E(G)}\left(\sqrt{d_{u}}+\sqrt{d_{v}}\right)^{2} \leq R(G) \leq-\frac{n}{2}+\frac{1}{2 \delta^{2}} \sum_{u v \in E(G)}\left(\sqrt{d_{u}}+\sqrt{d_{v}}\right)^{2} .
\end{gathered}
$$

Since

$$
\sum_{u v \in E(G)}\left(\sqrt{d_{u}}+\sqrt{d_{v}}\right)^{2}=\sum_{u v \in E(G)}\left(d_{u}+d_{v}\right)+2 \sum_{u v \in E(G)} \sqrt{d_{u} d_{v}}=M_{1}(G)+2 M_{2}^{1 / 2}(G),
$$


we obtain the third and forth inequalities.

If $G$ is a regular graph, then $\delta=\Delta$ and, in each line, the lower and upper bounds are the same, and they are equal to $R(G)$.

If the equality is attained in some bound, then we have either $d_{u} d_{v}=\delta^{2}$ for every $u v \in E(G)$ or $d_{u} d_{v}=\Delta^{2}$ for every $u v \in E(G)$. Thus, we have either $d_{u}=\delta$ for every $u \in V(G)$ or $d_{u}=\Delta$ for every $u \in V(G)$, and so, the graph is regular.

Proposition 7 has the following consequence on random graphs.

Corollary 8. In the Erdös-Rényi model $G(n, p)$, with $p \in[1 / 2,1)$ and $q=$ $1-p$, almost every graph $G$ satisfies

$$
\begin{aligned}
& q^{2} n^{2}+O\left(n^{3 / 2}(\log n)^{1 / 2}\right) \leq \frac{M_{1}(G)-2 M_{2}^{1 / 2}(G)}{n-2 R(G)} \leq p^{2} n^{2}+O\left(n^{3 / 2}(\log n)^{1 / 2}\right), \\
& q^{2} n^{2}+O\left(n^{3 / 2}(\log n)^{1 / 2}\right) \leq \frac{M_{1}(G)+2 M_{2}^{1 / 2}(G)}{n+2 R(G)} \leq p^{2} n^{2}+O\left(n^{3 / 2}(\log n)^{1 / 2}\right) .
\end{aligned}
$$

Proof. Proposition 7 gives

$$
\begin{aligned}
\delta^{2} & \leq \frac{M_{1}(G)-2 M_{2}^{1 / 2}(G)}{n-2 R(G)} \leq \Delta^{2}, \\
\delta^{2} & \leq \frac{M_{1}(G)+2 M_{2}^{1 / 2}(G)}{n+2 R(G)} \leq \Delta^{2} .
\end{aligned}
$$

Items (2) and (3) give for almost every graph

$$
\begin{aligned}
& \Delta^{2}=\left(p n+O\left((n \log n)^{1 / 2}\right)\right)^{2}=p^{2} n^{2}+O\left(n^{3 / 2}(\log n)^{1 / 2}\right), \\
& \delta^{2}=\left(q n+O\left((n \log n)^{1 / 2}\right)\right)^{2}=q^{2} n^{2}+O\left(n^{3 / 2}(\log n)^{1 / 2}\right) .
\end{aligned}
$$

These facts give the desired inequalities.

The following proposition is a consequence of (12) in [43].

Proposition 9. Let $G$ be a graph with $m$ edges, $n$ vertices, minimum degree $\delta$ and maximum degree $\Delta$. Then then

$$
R(G) \geq \frac{n}{2}-\frac{m}{2}\left(\frac{1}{\sqrt{\delta}}-\frac{1}{\sqrt{\Delta}}\right)^{2}
$$

and the equality is attained if and only if $G$ is a regular or biregular graph. 
Proof. Equation (12) can be written as

$$
R(G)=\frac{n}{2}-\frac{1}{2} \sum_{u v \in E(G)}\left(\frac{1}{\sqrt{d_{u}}}-\frac{1}{\sqrt{d_{v}}}\right)^{2},
$$

and so,

$$
R(G) \geq \frac{n}{2}-\frac{1}{2} \sum_{u v \in E(G)}\left(\frac{1}{\sqrt{\delta}}-\frac{1}{\sqrt{\Delta}}\right)^{2}=\frac{n}{2}-\frac{m}{2}\left(\frac{1}{\sqrt{\delta}}-\frac{1}{\sqrt{\Delta}}\right)^{2} .
$$

The equality is attained if and only if $\left\{d_{u}, d_{v}\right\}=\{\delta, \Delta\}$ for every $u v \in$ $E(G)$, i.e., $G$ is a regular or biregular graph.

Note that the lower bound in Proposition 9 is not comparable with the one in Corollary 5, as the following examples show:

If $G$ is the path graph with $n$ vertices, then

$$
\frac{n}{2}-\frac{m}{2}\left(\frac{1}{\sqrt{\delta}}-\frac{1}{\sqrt{\Delta}}\right)^{2}=\frac{n}{2}-\frac{n-1}{2}\left(1-\frac{1}{\sqrt{2}}\right)^{2} \approx \frac{2 \sqrt{2}+1}{4} n
$$

is larger than

$$
\frac{\sqrt{\Delta \delta}}{\Delta+\delta} n=\frac{\sqrt{2}}{3} n
$$

for large enough $n$. However, if $G$ is the complete graph with $n-1$ vertices $K_{n-1}$ with an additional edge joining a vertex of $K_{n-1}$ with an additional vertex of degree 1 , then

$$
\frac{\sqrt{\Delta \delta}}{\Delta+\delta} n=\frac{\sqrt{n-1}}{n} n=\sqrt{n-1}
$$

is larger than

$$
\frac{n}{2}-\frac{m}{2}\left(\frac{1}{\sqrt{\delta}}-\frac{1}{\sqrt{\Delta}}\right)^{2}=\frac{n}{2}-\frac{\frac{1}{2}(n-1)(n-2)+1}{2}\left(1-\frac{1}{\sqrt{n-1}}\right)^{2},
$$

for large enough $n$.

Proposition 10. In the Erdös-Rényi model $G(n, p)$, with $p \in[1 / 2,1)$ and $q=1-p$, almost every graph $G$ satisfies

$$
\begin{array}{ll}
R(G) \geq \frac{2 \sqrt{p q}+2 q-1}{4 q} n+o(n), & \text { if } \quad p>1 / 2, \\
R(G)=\frac{n}{2}+O\left((n \log n)^{1 / 2}\right), & \text { if } \quad p=1 / 2 .
\end{array}
$$


Proof. The second statement follows from Corollary 6 .

Assume now $p>1 / 2$. Items (2) and (3) give that in the Erdös-Rényi model $G(n, p)$, almost every graph $G$ satisfies

$$
\begin{aligned}
\left(\frac{1}{\sqrt{\delta}}-\frac{1}{\sqrt{\Delta}}\right)^{2} & =\frac{\Delta+\delta-2 \sqrt{\Delta \delta}}{\Delta \delta} \\
& =\frac{n+o\left((n \log n)^{1 / 2}\right)-2 \sqrt{p q n^{2}+O\left(n(n \log n)^{1 / 2}\right)}}{p q n^{2}+O\left(n(n \log n)^{1 / 2}\right)} \\
& =\frac{n+o\left((n \log n)^{1 / 2}\right)-2 \sqrt{p q} n+O\left((n \log n)^{1 / 2}\right)}{p q n^{2}+O\left(n(n \log n)^{1 / 2}\right)} \\
& =\frac{(1-2 \sqrt{p q}) n+O\left((n \log n)^{1 / 2}\right)}{p q n^{2}+O\left(n(n \log n)^{1 / 2}\right)} \\
& =\frac{1-2 \sqrt{p q}}{p q n}+o\left(\frac{1}{n}\right) .
\end{aligned}
$$

This fact, Proposition 9 and item (1) give

$$
\begin{aligned}
R(G) & \geq \frac{n}{2}-\frac{m}{2}\left(\frac{1}{\sqrt{\delta}}-\frac{1}{\sqrt{\Delta}}\right)^{2} \\
& =\frac{n}{2}-\frac{1}{2}\left(\frac{p n(n-1)}{2}+o\left(n^{2}\right)\right)\left(\frac{1-2 \sqrt{p q}}{p q n}+o\left(\frac{1}{n}\right)\right) \\
& =\frac{1}{2} n-\frac{1-2 \sqrt{p q}}{4 q} n+o(n)=\frac{2 \sqrt{p q}+2 q-1}{4 q} n+o(n) .
\end{aligned}
$$

The misbalance rodeg index is defined as

$$
M R(G)=\sum_{u v \in E(G)}\left|\sqrt{d_{u}}-\sqrt{d_{v}}\right| .
$$

This is a significant predictor of enthalpy of vaporization and of standard enthalpy of vaporization for octane isomers (see [44]).

Theorem 11. Let $G$ be a graph with maximum degree $\Delta$ and $m$ edges. Then

$$
R(G) \leq \frac{n}{2}-\frac{1}{2 \Delta^{2} m} M R(G)^{2}
$$

and the equality is attained if and only if $G$ is regular. 
Proof. By Cauchy-Schwarz inequality we have

$$
\begin{aligned}
M R(G)^{2} & =\left(\sum_{u v \in E(G)}\left|\sqrt{d_{u}}-\sqrt{d_{v}}\right|\right)^{2} \\
& \leq\left(\sum_{u v \in E(G)} 1^{2}\right) \sum_{u v \in E(G)}\left(\sqrt{d_{u}}-\sqrt{d_{v}}\right)^{2} \leq m \sum_{u v \in E(G)}\left(\sqrt{d_{u}}-\sqrt{d_{v}}\right)^{2} .
\end{aligned}
$$

Hence, (12) gives

$$
\begin{aligned}
R(G) & =\frac{n}{2}-\frac{1}{2} \sum_{u v \in E(G)} \frac{\left(\sqrt{d_{u}}-\sqrt{d_{v}}\right)^{2}}{d_{u} d_{v}} \\
& \leq \frac{n}{2}-\frac{1}{2 \Delta^{2}} \sum_{u v \in E(G)}\left(\sqrt{d_{u}}-\sqrt{d_{v}}\right)^{2} \leq \frac{n}{2}-\frac{1}{2 \Delta^{2} m} M R(G)^{2} .
\end{aligned}
$$

If $G$ is regular, then $R(G)=n / 2$ and $M R(G)=0$ and so, the equality is attained.

If the equality is attained, then $d_{u} d_{v}=\Delta^{2}$ for every $u v \in E(G)$; thus, $d_{u}=\Delta$ for all $u \in V(G)$ and so, $G$ is a regular graph.

Corollary 12. In the Erdös-Rényi model $G(n, p)$, with $p \in[1 / 2,1)$ and $q=$ $1-p$, almost every graph $G$ satisfies

$$
\frac{M R(G)^{2}}{n-2 R(G)} \leq \frac{1}{2} p^{2} n^{3}+o\left(n^{3}\right)
$$

Proof. Theorem 11 gives the inequality

$$
\frac{M R(G)^{2}}{n-2 R(G)} \leq \Delta^{2} m .
$$

Items (1) and (2) give that in the Erdös-Rényi model $G(n, p)$, almost every graph $G$ satisfies

$$
\begin{aligned}
\Delta^{2} m & =\left(p n+O\left((n \log n)^{1 / 2}\right)\right)^{2}\left(\frac{p n(n-1)}{2}+o\left(n^{2}\right)\right) \\
& =\frac{1}{2} p^{2} n^{3}+o\left(n^{3}\right),
\end{aligned}
$$

and this gives the desired inequality.

The following Szökefalvi Nagy inequality appears in [45] (see also [46]). 
Lemma 13. If $a_{j} \geq 0$ for $1 \leq j \leq k, R=\max _{j} a_{j}$ and $r=\min _{j} a_{j}$, then

$$
k \sum_{j=1}^{k} a_{j}^{2}-\left(\sum_{j=1}^{k} a_{j}\right)^{2} \geq \frac{k}{2}(R-r)^{2} .
$$

In many papers the hypothesis $a_{j} \geq 0$ for $1 \leq j \leq k, R=\max _{j} a_{j}$ and $r=\min _{j} a_{j}$, is replaced by $0<r \leq a_{j} \leq R$ for $1 \leq j \leq k$. However, the conclusion of Lemma 13 does not hold in general with the hypothesis $0<r \leq a_{j} \leq R$ for $1 \leq j \leq k$, as the following example shows:

If $a_{j}=a$ for $1 \leq j \leq k, R>a$ and $r \leq a<R$, then

$$
k \sum_{j=1}^{k} a_{j}^{2}-\left(\sum_{j=1}^{k} a_{j}\right)^{2}=k^{2} a^{2}-k^{2} a^{2}=0<\frac{k}{2}(R-r)^{2} .
$$

Theorem 14. Let $G$ be a graph with $m$ edges,

$$
\Pi=\max _{u v \in E(G)} \frac{1}{\sqrt{d_{u} d_{v}}}, \quad \text { and } \quad \pi=\min _{u v \in E(G)} \frac{1}{\sqrt{d_{u} d_{v}}} .
$$

Then

$$
R(G) \leq \sqrt{m M_{2}^{-1}(G)-\frac{m}{2}(\Pi-\pi)^{2}},
$$

and the equality is attained if $G$ is a regular or biregular graph.

Proof. If we choose $a_{j}=1 / \sqrt{d_{u} d_{v}}$, Lemma 13 gives

$$
\begin{aligned}
m M_{2}^{-1}(G)-R(G)^{2} & =m \sum_{u v \in E(G)} \frac{1}{d_{u} d_{v}}-\left(\sum_{u v \in E(G)} \frac{1}{\sqrt{d_{u} d_{v}}}\right)^{2} \\
& \geq \frac{m}{2}(\Pi-\pi)^{2},
\end{aligned}
$$

and this gives the inequality.

If $G$ is a biregular or regular graph, then

$$
\frac{1}{\sqrt{d_{u} d_{v}}}=\frac{1}{\sqrt{\Delta \delta}}=\Pi=\pi
$$

for every $u v \in E(G)$. Thus,

$$
\sqrt{m M_{2}^{-1}(G)-\frac{m}{2}(P-p)^{2}}=\sqrt{m \frac{m}{\Delta \delta}}=\frac{m}{\sqrt{\Delta \delta}}=R(G) .
$$


The inverse degree index $I D(G)$ is defined by

$$
I D(G)=\sum_{u \in V(G)} \frac{1}{d_{u}}=\sum_{u v \in E(G)}\left(\frac{1}{d_{u}^{2}}+\frac{1}{d_{v}^{2}}\right)=\sum_{u v \in E(G)} \frac{d_{u}^{2}+d_{v}^{2}}{d_{u}^{2} d_{v}^{2}}
$$

The inverse degree index of a graph has been studied by several authors (see, e.g., [47, 48, 49] and the references therein). The following result provides some inequalities relating Randić and Inverse Degree indices (see [50] for other inequalities relating these indices).

Theorem 15. Let $G$ be a graph with minimum degree $\delta$ and maximum degree $\Delta$. Then

$$
\begin{array}{ll}
\frac{\delta}{2} I D(G) \leq R(G) \leq \frac{\Delta}{2} I D(G), & \text { if } \delta \geq s_{0} \Delta, \\
\frac{(\Delta \delta)^{3 / 2}}{\Delta^{2}+\delta^{2}} I D(G) \leq R(G) \leq \frac{\Delta}{2} I D(G), & \text { if } \delta \leq s_{0} \Delta,
\end{array}
$$

where $s_{0}$ is the unique solution of the equation $s^{2}-2 \sqrt{s}+1=0$ in $(0,1)$. Furthermore, the upper bound is attained if and only if $G$ is regular; if $\delta \geq$ $s_{0} \Delta$, then the lower bound is attained if and only if $G$ is regular; if $\delta \leq s_{0} \Delta$, then the lower bound is attained if and only if $G$ is biregular.

Proof. First of all, let us check that $s_{0}$ is well-defined, i.e., there exists a unique solution of the equation $s^{2}-2 \sqrt{s}+1=0$ in $(0,1)$. By making the change of variable $s=t^{2}$, we see that this holds if and only if there exists a unique solution of the equation $t^{4}-2 t+1=0$ in $(0,1)$. Note that $t^{4}-2 t+1=(t-1) u(t)$, with $u(t)=t^{3}+t^{2}+t-1$. Since $u(0)=-1, u(1)=2$ and $u^{\prime}(t)=3 t^{2}+2 t+1>0$ on $(0,1)$, we conclude that there is a unique zero $t_{0}$ of $u$ in $(0,1)$ and, in fact, $u(t)<0$ for every $t \in\left(0, t_{0}\right)$ and $u(t)>0$ for every $t \in\left(t_{0}, 1\right)$. If $s_{0}=t_{0}^{2}$, then $s^{2}-2 \sqrt{s}+1>0$ for $s \in\left(0, s_{0}\right)$ and $s^{2}-2 \sqrt{s}+1<0$ for every $s \in\left(s_{0}, 1\right)$.

Let $f:[\delta, \Delta] \times[\delta, \Delta] \rightarrow \mathbb{R}$ be the function given by

$$
f(x, y)=\left(\frac{1}{x^{2}}+\frac{1}{y^{2}}\right) \sqrt{x y}=x^{-3 / 2} y^{1 / 2}+y^{-3 / 2} x^{1 / 2} .
$$


First we will find the minimum and maximum values of $f$. We can assume that $x \leq y$ (symmetry).

$$
\frac{\partial f}{\partial x}(x, y)=-\frac{3}{2} x^{-5 / 2} y^{1 / 2}+\frac{1}{2} x^{-1 / 2} y^{-3 / 2}=\frac{1}{2} x^{-5 / 2} y^{-3 / 2}\left(x^{2}-3 y^{2}\right) .
$$

Thus,

$$
\frac{\partial f}{\partial x}(x, y)<0, \quad \text { if } \delta \leq x \leq y \leq \Delta,
$$

and so, the function $f$ attains its maximum value in the set $\{x=\delta, \delta \leq y \leq$ $\Delta\}$, and the minimum value in the set $\{\delta \leq x=y \leq \Delta\}$. Thus,

$$
\begin{aligned}
f(x, y) & \geq \min _{\delta \leq x \leq \Delta} f(x, x)=\min _{\delta \leq x \leq \Delta} \frac{2}{x^{2}} x=\frac{2}{\Delta}, \\
\frac{1}{d_{u}^{2}}+\frac{1}{d_{v}^{2}} & \geq \frac{2}{\Delta} \frac{1}{\sqrt{d_{u} d_{v}}}, \\
R(G) & \leq \frac{\Delta}{2} I D(G) .
\end{aligned}
$$

Since

$$
\frac{\partial f}{\partial y}(x, y)=\frac{1}{2} y^{-5 / 2} x^{-3 / 2}\left(y^{2}-3 x^{2}\right),
$$

if $\Delta^{2}-3 \delta^{2}<0$, then

$$
\frac{\partial f}{\partial y}(\delta, y)=\frac{1}{2} y^{-5 / 2} \delta^{-3 / 2}\left(y^{2}-3 \delta^{2}\right) \leq \frac{1}{2} y^{-5 / 2} \delta^{-3 / 2}\left(\Delta^{2}-3 \delta^{2}\right)<0,
$$

and

$$
f(x, y) \leq \max _{\delta \leq y \leq \Delta} f(\delta, y)=f(\delta, \delta)=\frac{2}{\delta} .
$$

If $\Delta^{2}-3 \delta^{2} \geq 0$, then

$$
\frac{\partial f}{\partial y}(\delta, y)=\frac{1}{2} y^{-5 / 2} \delta^{-3 / 2}\left(y^{2}-3 \delta^{2}\right) \leq 0
$$

if and only if $y \in[\delta, \sqrt{3} \delta]$. Thus, $f(\delta, y)$ decreases on $[\delta, \sqrt{3} \delta]$ and increases on $[\sqrt{3} \delta, \Delta]$. Hence, we have in both cases

$f(x, y) \leq \max _{\delta \leq y \leq \Delta} f(\delta, y)=\max \{f(\delta, \delta), f(\delta, \Delta)\}=\max \left\{\frac{2}{\delta},\left(\frac{1}{\delta^{2}}+\frac{1}{\Delta^{2}}\right) \sqrt{\delta \Delta}\right\}$. 
Recall that $s^{2}-2 \sqrt{s}+1>0$ on $\left(0, s_{0}\right)$. Thus, we have for $\delta \leq s_{0} \Delta$,

$$
\left(1+\frac{\delta^{2}}{\Delta^{2}}\right) \geq 2 \sqrt{\frac{\delta}{\Delta}}, \quad\left(\frac{1}{\delta^{2}}+\frac{1}{\Delta^{2}}\right) \sqrt{\delta \Delta} \geq \frac{2}{\delta},
$$

and we conclude

$$
\begin{aligned}
f(x, y) & \leq \max \left\{\frac{2}{\delta},\left(\frac{1}{\delta^{2}}+\frac{1}{\Delta^{2}}\right) \sqrt{\delta \Delta}\right\}=\frac{\Delta^{2}+\delta^{2}}{(\Delta \delta)^{3 / 2}} \\
\frac{1}{d_{u}^{2}}+\frac{1}{d_{v}^{2}} & \leq \frac{\Delta^{2}+\delta^{2}}{(\Delta \delta)^{3 / 2}} \frac{1}{\sqrt{d_{u} d_{v}}}, \\
R(G) & \geq \frac{(\Delta \delta)^{3 / 2}}{\Delta^{2}+\delta^{2}} I D(G) .
\end{aligned}
$$

If $\delta \geq s_{0} \Delta$, then $f(x, y) \leq f(\delta, \delta)=2 / \delta$ and

$$
R(G) \geq \frac{\delta}{2} I D(G)
$$

The previous argument gives that the upper bound is attained if and only if $d_{u}=d_{v}=\Delta$ for every $u v \in E(G)$, and this happens if and only if $G$ is regular.

Assume that $\delta \geq s_{0} \Delta$. Thus, the lower bound is attained if and only if $d_{u}=d_{v}=\delta$ for every $u v \in E(G)$, i.e., if and only if $G$ is regular.

Assume that $\delta \leq s_{0} \Delta$. Thus, the lower bound is attained if and only if $\left\{d_{u}, d_{v}\right\}=\{\Delta, \delta\}$ for every $u v \in E(G)$, i.e., if and only if $G$ is biregular (note that $G$ can not be a regular graph since $\delta \leq s_{0} \Delta<\Delta$ ).

Theorem 15 has the following consequence on random graphs.

Corollary 16. In the Erdös-Rényi model $G(n, p)$, with $p \in[1 / 2,1)$ and $q=$ $1-p$, almost every graph $G$ satisfies

$$
\min \left\{\frac{q}{2}, \frac{(p q)^{3 / 2}}{p^{2}+q^{2}}\right\} n+O\left((n \log n)^{1 / 2}\right) \leq \frac{R(G)}{I D(G)} \leq \frac{p}{2} n+O\left((n \log n)^{1 / 2}\right) .
$$

Proof. Theorem [15] can be stated as follows:

$$
\min \left\{\frac{\delta}{2}, \frac{(\Delta \delta)^{3 / 2}}{\Delta^{2}+\delta^{2}}\right\} \leq \frac{R(G)}{I D(G)} \leq \frac{\Delta}{2} .
$$


Items (2) and (3) give for almost every graph

$$
\begin{aligned}
\frac{(\Delta \delta)^{3 / 2}}{\Delta^{2}+\delta^{2}} & =\frac{\left(p q n^{2}+O\left(n(n \log n)^{1 / 2}\right)\right)^{3 / 2}}{\left(p^{2}+q^{2}\right) n^{2}+O\left(n(n \log n)^{1 / 2}\right)}=\frac{(p q)^{3 / 2} n^{3}\left(1+\frac{3}{2} \frac{O\left(n(n \log n)^{1 / 2}\right)}{p q n^{2}}\right)}{\left(p^{2}+q^{2}\right) n^{2}+O\left(n(n \log n)^{1 / 2}\right)} \\
& =\frac{O\left(n^{2}(n \log n)^{1 / 2}\right)}{\left(p^{2}+q^{2}\right) n^{2}+O\left(n(n \log n)^{1 / 2}\right)}+\frac{\left.O(n \log n)^{1 / 2}\right)}{\left(p^{2}+q^{2}\right) n^{2}+O\left(n(n \log n)^{1 / 2}\right)} \\
& =\frac{(p q)^{3 / 2} n}{p^{2}+q^{2}}\left(1-\frac{O\left((n \log n)^{1 / 2}\right)}{}\right. \\
& =\frac{(p q)^{3 / 2}}{p^{2}+q^{2}} n+O\left((n \log n)^{1 / 2}\right) .
\end{aligned}
$$

These facts, and items (2) and (3) give for almost every graph

$$
\begin{array}{r}
\min \left\{\frac{q}{2} n+O\left((n \log n)^{1 / 2}\right), \frac{(p q)^{3 / 2}}{p^{2}+q^{2}} n+O\left((n \log n)^{1 / 2}\right)\right\} \leq \frac{H(G)}{I D(G)} \\
\leq \frac{p}{2} n+O\left((n \log n)^{1 / 2}\right)
\end{array}
$$

and this finishes the proof.

\section{Summary}

Based on the important theoretical-practical applications of the Randic index, in this paper we have studied computationally and analytically the properties of the Randić index $R(G)$ in Erdös-Rényi graphs $G(n, p)$ characterized by $n$ vertices connected independently with probability $p \in(0,1)$.

First, by the proper scaling analysis of the average (and normalized) Randić index, $\langle\bar{R}(G)\rangle=\langle R(G)\rangle /(n / 2)$, we found that $\xi \approx n p$ is the scaling parameter of $R(G(n, p))$; that is, for fixed $\xi,\langle\bar{R}(G)\rangle$ is also fixed, see Fig. 2(b). Moreover, our analysis provides a way to predict the value of the Randić index on Erdös-Rényi graphs once the value of $\xi$ is known: $R(G) \approx 0$ for $\xi<0.01$ (when the vertices in the graph are mostly isolated), the transition from isolated vertices to complete graphs occurs in the interval $0.01<\xi<10$ where $0<R(G)<n / 2$, while when $\xi>10$ the graphs are almost complete and $R(G) \approx n / 2$. These intervals are indicated as verti-

cal dashed lines in Fig. 2(b). Also, to extend the applicability of our scaling analysis we demonstrate that for fixed $\xi$ the spectral properties of $R(G(n, p))$ (characterized by the energy of the corresponding Randic matrix) are also 
universal; i.e., they do not depend on the specific values of the individual graph parameters, see Fig. 3(c).

In particular, we would like to stress that here we have successfully introduced a scaling approach to the study of topological indexes.

Then, to complement the study of the Randic index we have explored the relations between $R(G)$ and other important topological indexes such as the (-2) sum-connectivity index, the misbalance rodeg index, the inverse degree index, among others. In particular, we characterized graphs which are extremal with respect to those relations.

\section{Acknowledgements}

C.T.M.-M. and J.A.M.-B. thank partial support by VIEP-BUAP (Grant No. MEBJ-EXC18-G), Fondo Institucional PIFCA (Grant No. BUAP-CA169), and CONACyT (Grant No. CB-2013/220624), Mexico. J.M.R. and J.M.S. were supported in part by two grants from Ministerio de Economía y Competitividad, Agencia Estatal de Investigaciǿn (AEI) and Fondo Europeo de Desarrollo Regional (FEDER) (MTM2016-78227-C2-1-P and MTM201790584-REDT), Spain.

\section{References}

\section{References}

[1] H. Wiener, Structural determination of paraffin boiling points, J. Am. Chem. Soc. 69, 17 (1947).

[2] M. Randić, On characterization of molecular branching, J. Am. Chem. Soc. 97, 6609 (1975).

[3] I. Gutman and B. Furtula (eds.), Recent results in the theory of Randić index (Univ. Kragujevac, Kragujevac, 2008).

[4] X. Li and I. Gutman, Mathematical aspects of Randić type molecular structure descriptors (Univ. Kragujevac, Kragujevac, 2006).

[5] X. Li and Y. Shi, A survey on the Randić index, MATCH Commun. Math. Comput. Chem. 59, 127 (2008).

[6] X. Li and J. Zheng, A unified approach to the extremal trees for different indices, MATCH Commun. Math. Comput. Chem. 54, 195 (2005). 
[7] X. Li and H. Zhao, Trees with the first smallest and largest generalized topological indices, MATCH Commun. Math. Comput. Chem. 50, 57 (2004).

[8] A. Miličević and S. Nikolić, On variable Zagreb indices, Croat. Chem. Acta 77, 97 (2004).

[9] M. Randić, Novel graph theoretical approach to heteroatoms in quantitative structure-activity relationships, Chemometrics Intel. Lab. Syst. 10, 213 (1991).

[10] M. Randić, On computation of optimal parameters for multivariate analysis of structure-property relationship, J. Chem. Inf. Comput. Sci. 31, 970 (1991).

[11] S. Nikolić, A. Miličević , N. Trinajstić and A. Jurić, On use of the variable Zagreb ${ }^{\nu} M_{2}$ Index in QSPR: Boiling points of Benzenoid hydrocarbons, Molecules 9, 1208 (2004).

[12] B. Zhou and N. Trinajstić, On general sum-connectivity index, J. Math. Chem. 47, 210 (2010).

[13] J. M. Rodríguez and J. M. Sigarreta, New results on the Harmonic index and its generalizations, MATCH Commun. Math. Comput. Chem. 78, 387 (2017).

[14] I. Gutman, B. Furtula and V. Katanić, Randić index and information, AKCE Int. J. Graphs Comb. in press, (2018).

[15] N. Nikolova and J. Jaworska, Approaches to measure chemical similaritya review, QSAR Comb. Sci. 22, 1006 (2003).

[16] M. Randić, On the history of the connectivity index: from the connectivity index to the exact solution of the protein alignment problem, SAR QSAR Environ. Res. 26, 523 (2015).

[17] E. Estrada, Quantifying network heterogeneity, Phys Rev. E 82, 066102 (2010).

[18] P. de Meo, F. Messina, D. Rosaci, G. M. L. Sarné and A. V. Vasilakos, Estimating graph robustness through the Randic index, IEEE Trans. Cybern. 99, 1 (2017). 
[19] Z. Chen, M. Dehmer, F. Emmert-Streib and Y. Shi, Entropy of weighted graphs with Randić weights, Entropy 17, 3710 (2015).

[20] L. da F. Costa, O. N. Oliveira Jr, G. Travieso, F. A. Rodrigues, P. R. Villas Boas, L. Antiqueira, M. P. Viana, and L. E. C. Rocha, Analyzing and modeling real-world phenomena with complex networks: a survey of applications, Advances in Physics 60, 329 (2011).

[21] A. L. Barabasi, Network science, Phil. Trans. R. Soc. A 371, 20120375 (2013).

[22] O. Mülken and A. Blumen, Continuous-time quantum walks: Models for coherent transport on complex networks, Phys. Rep. 502, 37 (2011).

[23] M. E. J. Newman, Networks: An introduction (Oxford University Press, New York, 2010).

[24] S. Boccaletti, V. Latora, Y. Moreno, M. Chavez, and D.-U. Hwang, Complex networks: Structure and dynamics, Phys. Rep. 424, 175 (2006).

[25] B. Bollobás, Random Graphs in Modern Graph Theory, Graduate Texts in Mathematics Volume 184, pp 215-252 (Springer, New York, 1998).

[26] R. Solomonoff and A. Rapoport, Connectivity of random nets, Bull. Math. Biophys. 13, 107 (1951).

[27] P. Erdős and A. Rényi, On Random Graphs, Publ. Math. (Debrecen) 6, 290 (1959).

[28] P. Erdős and A. Rényi, On the evolution of random graphs, Inst. of the Hung. Acad. of Sci. 5, 17 (1960); On the strength of connectedness of a random graph, Acta Mathematica Hungarica 12, 261 (1961).

[29] J. A. Mendez-Bermudez, A. Alcazar-Lopez , A. J. Martinez-Mendoza, F. A. Rodrigues and T. K. DM. Peron, Universality in the spectral and eigenfunction properties of random networks, Phys. Rev. E 91, 032122 (2015).

[30] A. J. Martinez-Mendoza, A. Alcazar-Lopez and J. A Mendez-Bermudez, Scattering and transport properties of tight-binding random networks, Phys. Rev. E 88, 122126 (2013). 
[31] R. Gera, L. Alonso, B. Crawford, J. House, J. A. Mendez-Bermudez, T. Knuth and R. Miller, Identifying network structure similarity using spectral graph theory, Appl. Net. Sci. 3, 2 (2018).

[32] C. T. Martinez-Martinez and J. A. Mendez-Bermudez, Information entropy of tight-binding random networks with losses and gain: Scaling and universality, Entropy 21, 86 (2019).

[33] G. Torres-Vargas, R. Fossion, and J. A. Mendez-Bermudez Normal mode analysis of spectra of random networks, submitted (2019).

[34] J. A. Rodriguez and J. M Sigarreta, On the Randić index and conditional parameters of a graph, MATCH Commun. Math. Comput. Chem. 54, 403 (2005).

[35] S. B. Bozkurt, A. D. Güngör, I. Gutman and A. S. Cevik, Randić matrix and Randić energy, MATCH Commun. Math. Comput. Chem. 64, 239 (2010).

[36] A. D. Mirlin and Y. V. Fyodorov, Universality of level correlation function of sparse random matrices, J. Phys. A: Math. Gen. 24, 2273 (1991).

[37] Y. V. Fyodorov and A. D. Mirlin, Localization in ensemble of sparse random matrices, Phys. Rev. Lett. 67, 2049 (1991).

[38] S. N. Evangelou and E. N. Economou, Spectral density singularities, level statistics, and localization in a sparse random matrix ensemble, Phys. Rev. Lett. 68, 361 (1992).

[39] S. N. Evangelou, A numerical study of sparse random matrices, J. Stat. Phys. 69, 361 (1992).

[40] B. Bollobás, Degree sequences of random graphs, Discrete Math. 33, 1 (1981).

[41] C. Dalfó, On the Randić index of graphs, Discrete Math. in press, (2018).

[42] J. M. Rodriguez and J. M. Sigarreta, On the Geometric-Arithmetic index, MATCH Commun. Math. Comput. Chem. 74, 103 (2015). 
[43] K. C. Das, S. Balachandran and I. Gutman, Inverse degree, Randić index and harmonic index of graphs, Appl. Anal. Discrete Math. 11, 304 (2017).

[44] D. Vukičević and M. Gašperov, Bond additive modeling 1. Adriatic indices, Croat. Chem. Acta 83, 243 (2010).

[45] J. S. Nagy, Uber algebraische Gleichungen mit lauter reellenWurzeln, Jahresbericht der Deutschen mathematiker-Vereingung 27, 37 (1918).

[46] R. Sharma, M. Gupta and G. Kopor, Some better bounds on the variance with applications, J. Math. Ineq. 4, 355 (2010).

[47] P. Dankelmann, A. Hellwig and L. Volkmann, Inverse degree and edgeconnectivity, Discrete Math. 309, 2943 (2008).

[48] K.C. Das, K. Xu and J. Wang, On inverse degree and topological indices of graphs, Filomat 30, 2111 (2016).

[49] P. Erdös, J. Pach and J. Spencer, On the mean distance between points of a graph, Congr. Numer. 64, 121 (1988).

[50] J. M. Rodríguez, J. L. Sánchez and J. M. Sigarreta, Inequalities on the inverse degree index, submitted (2018). 